\title{
Using Ambient Vibration Measurements (AVM) and Operational Modal Analysis (OMA) to Characterize Telecommunication Monopoles
} \author{
Savreux ${ }^{5}$ and Simon Humeau ${ }^{6}$ \\ ${ }^{1}$ Professor, Department of Civil Engineering and Applied Mechanics, McGill University, Canada \\ ${ }^{2,3}$ Department of Civil Engineering and Applied Mechanics, McGill University, Canada \\ ${ }^{4,5,6}$ McGill University visiting students from University of Angers, France
}

Ghyslaine McClure ${ }^{1 *}$, Samuel Jubinville-Baron ${ }^{2}$, Jordann Jubinville-Baron ${ }^{3}$, Antoine Martineau ${ }^{4}$, Louis

*Corresponding author: Ghyslaine McClure, Department of Civil Engineering and Applied Mechanics, McGill University, Montréal, H3A 0C3, Canada.

Received Date: August 09, 2019

Published Date: September 03, 2019

Abstract

This paper presents the dynamic characteristics of 20 steel telecommunications monopoles as extracted from ambient vibration measurements made directly on the base and on the structure itself at low height. Velocities are measured during a minimum of 30 minutes at each site with highly sensitive micrometers, synchronously at the different positions. The records are analysed using a frequency domain decomposition algorithm available in commercial software. Statistical analysis of the extracted results indicate that the fundamental natural frequencies could be identified with high precision (standard deviation less than $4 \%$ ) while the corresponding total equivalent damping was less precise, with standard deviations between 6 and 28\%. The low-cost, non-intrusive AVM tests are deemed appropriate to evaluate the vulnerability of telecommunications monopoles to wind-induced instabilities.

\section{Introduction}

This experimental study is motivated by the need for telecommunications monopoles designers to assess the vulnerability of tall and smooth structures to wind-induced instabilities such as vortex shedding and flutter. Recent editions of North American telecommunication structures design codes, such as CSA S37-18 [1] in Canada and ANSI/TIA-222-H [2] in the US have provided guidelines to address fatigue in poles (monopoles and tripoles, in particular) that preclude the knowledge of their modal damping in the fundamental mode. Also, the evaluation of the critical wind velocity for vortex shedding requires the knowledge of the fundamental or first few lower natural frequencies of the structures. These dynamic properties are difficult to predict by calculations and in situ ambient vibration measurements (AVM) provide the ideal way to determine them while taking into account the effects of the foundation, antennas and all attached appurtenances.

\section{Tested Structures and Protocol}

With the collaboration of the owners and their engineering consultant, it was possible to conduct AVM tests at 20 cellular telecommunications monopoles sites located in Québec, Canada, between 10 May and 12 July 2019. Site No. 16 is shown as an example in Figure 1A. The structures vary in height from $21,3 \mathrm{~m}$ to $50,9 \mathrm{~m}$. Most of them are made of smooth circular tubes while a few are multi-sided. Although their detailed geometric/mass characteristics cannot be disclosed, their dimensions varied from approximately $600-1800 \mathrm{~mm}$ width and 6-12 $\mathrm{mm}$ thickness at the base and 400-1300 mm width and 5-13 mm thickness at the top. Three types of joints are used: bolted, welded and section overlaps. The sensors are Tromino 3G ENGY micrometers [3] that measure the velocity of the structure to an accuracy of 10-6 m/s $(\mu \mathrm{m} / \mathrm{s})$. Sites Nos. 1 to 11 have used two sensors, one placed on the concrete base and another attached to the structure using a kevlar sling system. Sites Nos. 12 to 20 have used a second sensor on the structure using a magnet support system, as shown in Figure 1B. In all most cases two 30-minute long records were collected in three orthogonal directions; only one test was performed at the last tested sites as the preliminary analysis of the results indicated good coherence and accuracy. The measurements of the sensors were all synchronized using a wireless antenna. The wind speed at the site 
was measured using an EXTECH Mini Thermo-Anemometer 45158, base of the monopole was sheltered from wind or the wind speed at a height of approximately $2 \mathrm{~m}$ above ground. In some cases, the was too low to be measured (Figure 1A,1B).

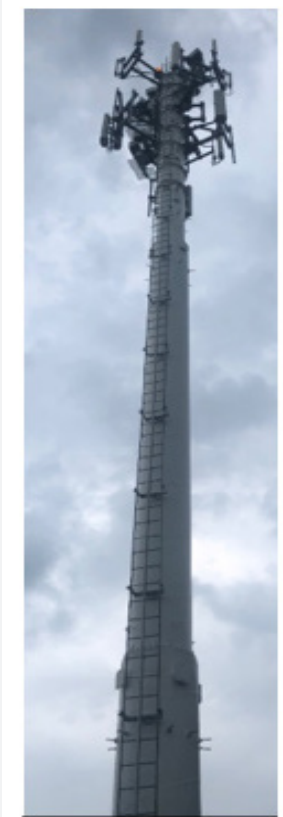

(a)

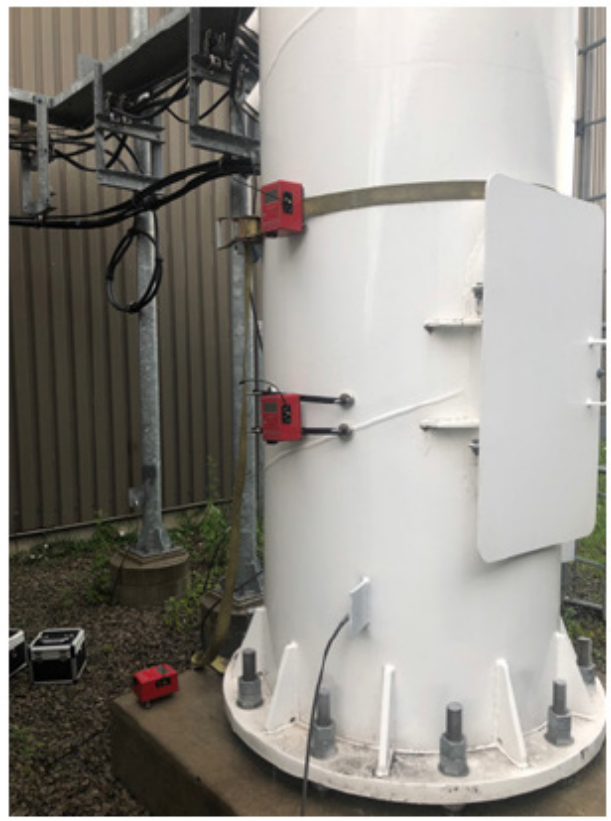

(b)

Figure 1: Tested monopole at Site 16 (a) with instrumentation in place (b).

\section{Operational Modal Analysis}

Once the in-situ data acquisition is completed, the recorded 30-min velocity time histories are transferred from the individual TROMINO sensors to a personal computer where they can be analysed by the ARTeMIS Modal Standard Software [4]. This software allows the identification of the modal frequencies of the structure using the Enhanced Frequency Domain Decomposition (EFDD) Method as well as the approximate global damping corresponding to each identified mode. An example of ARTeMIS data extraction window is shown in Figure 2 for a single record at Site No. 16 (Figure 2).

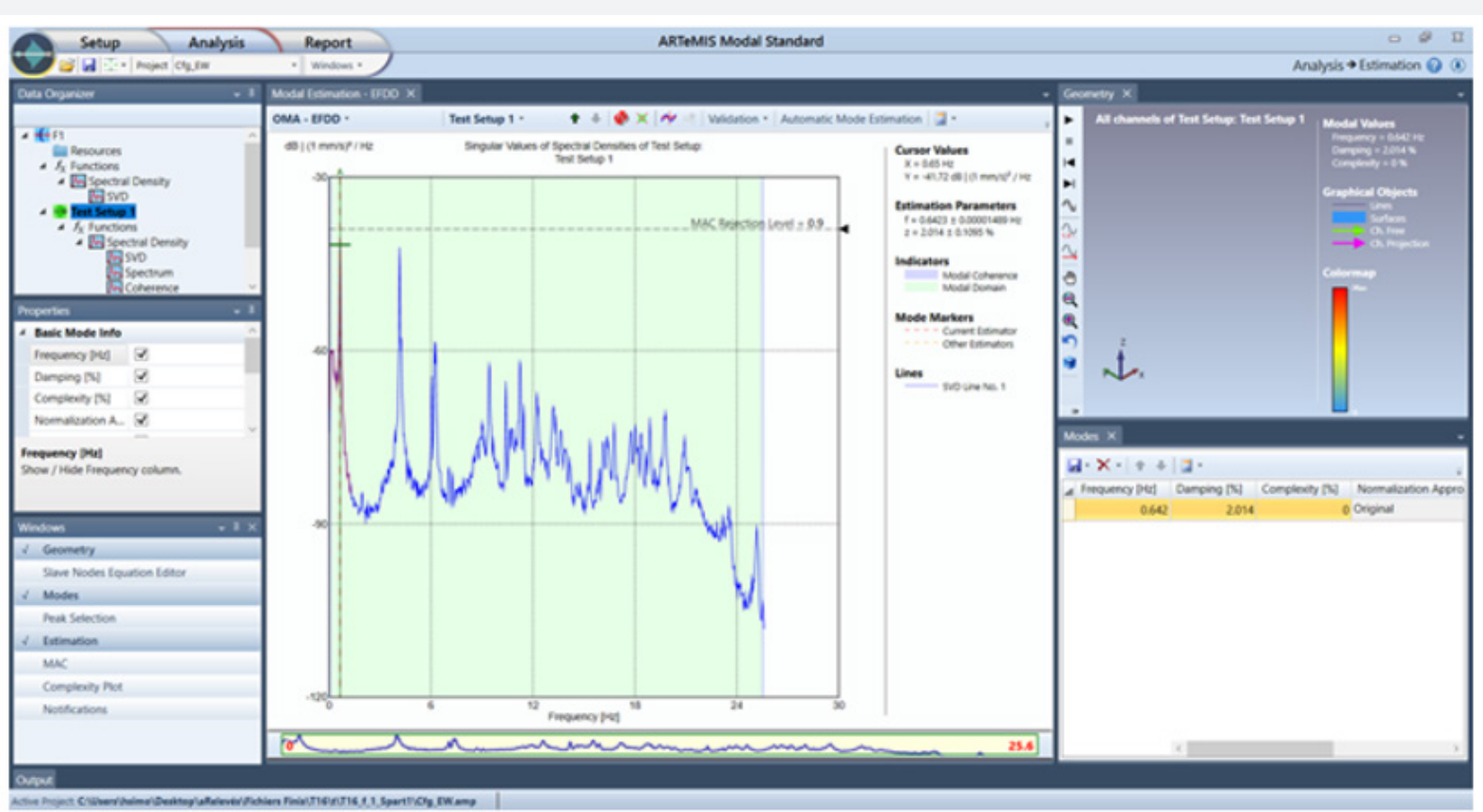

Figure 2: Natural frequency and equivalent damping extraction in ARTeMIS software for Site 16.

The EFDD method uses a peak-picking algorithm in the frequency domain to select resonant peaks. The peaks selected are not single values yet, but a band of the width of the peak. This band is identified with the modal assurance criterion (MAC), 
which poses a boundary of acceptance of the peaks. The MAC value varies between zero, representing a non-consistent mode shape, and one, an entirely consistent one. [5] A default MAC value of 0.9 was used for this study, which yielded consistent results. This MAC value ensures that the automatically identified peak is one of real resonance. A power spectral density function is then defined around the identified peak and used to obtain the frequency, through a trip back to the time domain, to obtain the zero crossing times. The damping ratio estimates are obtained through logarithmic decrement of the correlation time functions corresponding to each natural frequency identified. [6] The resulting fundamental frequencies and equivalent damping ratios are the two indicators that are the focus of this study. The EFDD method was used a number of times in different software, showing its high reliability for frequency extraction. [7] However, damping ratio estimates have larger uncertainty, in the order of $20 \%$, and better estimates are found for longer measurement samples. [8] The uncertainty in the damping estimation method is mainly attributed to the nonproportionality of damping observed in real structures, as opposed to the classical logarithmic decrement assumption associated with the ideal linear viscous damping model.

\section{Results and Observations}

Table 1 summarizes the results obtained for all tested sites. The values reported are extracted from the whole 30-min records while the standard deviations on these values are obtained by analysis of shorter sub-sets of 1-min duration. One would expect variability as the ambient vibrations, mostly caused by wind, vary naturally during the test period. It is seen form the table that the fundamental frequencies of the monopoles can be obtained with much smaller variability (from $0.5 \%$ to $3.3 \%$ ) than for the corresponding damping ratios, with standard deviations in the 6-28\% range. This is expected as shown by Magalhães et al. [8] who concluded than damping values determined from relatively long records are necessary to provide reliable measures. The fundamental frequencies of the tested monopoles vary in the range of 0,3 to $1,4 \mathrm{~Hz}$, depending on the lateral stiffness and reactive mass of the different structures. To cover the extreme cases, at site No. 14, the structure is very flexible (O.D. max. is $610 \mathrm{~mm}$ with $11 \mathrm{~mm}$ thickness, height 33,5 m) and supports a large number of antennas and accessories, while at site No. 4 , the bending rigidity of the structure is relatively large (O.D. max. $1360 \mathrm{~mm}$ and O.D. min. $1000 \mathrm{~mm}$ with 9,5 mm thickness, height 25,1 m) and lightweight antennas.

Table 1: Summary of fundamental frequencies and net damping obtained from OMA.

\begin{tabular}{|c|c|c|c|c|c|c|c|c|c|c|}
\hline \multirow{2}{*}{ Site No. } & \multirow{2}{*}{$\mathrm{H}(\mathrm{m})$} & \multirow{2}{*}{ f1 $(\mathrm{Hz})$} & \multicolumn{2}{|c|}{ Standard Deviation of $f 1$} & \multirow[t]{2}{*}{ ६1 (\%) } & \multicolumn{2}{|c|}{ Standard Deviation of $\xi 1$} & \multicolumn{3}{|c|}{ Connection Types ( ${ }^{*}$ ) } \\
\hline & & & $(\mathrm{Hz})$ & $\%$ f1 & & $(\%)$ & $\% \xi 1$ & B & 0 & w \\
\hline 1 & 36,6 & 0,48 & 0,007 & $1,5 \%$ & 2,8 & 0,45 & $16 \%$ & 0 & 3 & 1 \\
\hline 2 & 28,3 & 1,27 & 0,014 & $1,1 \%$ & 1,2 & 0,34 & $28 \%$ & 0 & 3 & 0 \\
\hline 3 & 48,9 & 0,54 & 0,018 & $3,3 \%$ & 4,1 & 0,85 & $21 \%$ & 3 & 0 & 0 \\
\hline 4 & 25,1 & 1,37 & 0,010 & $0,7 \%$ & 1,0 & 0,22 & $22 \%$ & 0 & 1 & 1 \\
\hline 5 & 36,7 & 0,69 & 0,023 & $3,3 \%$ & 2,0 & 0,28 & $14 \%$ & 0 & 1 & 2 \\
\hline 6 & 35,1 & 0,53 & 0,008 & $1,5 \%$ & 2,7 & 0,31 & $11 \%$ & 2 & 0 & 0 \\
\hline 7 & 21,3 & 0,32 & 0,008 & $2,5 \%$ & 4,7 & 0,47 & $10 \%$ & 1 & 0 & 0 \\
\hline 8 & 30,7 & 0,84 & 0,014 & $1,7 \%$ & 2,1 & 0,52 & $25 \%$ & 0 & 2 & 0 \\
\hline 9 & 30,1 & 0,67 & 0,007 & $1,0 \%$ & 2,1 & 0,34 & $16 \%$ & 2 & 0 & 0 \\
\hline 10 & 36,6 & 0,66 & 0,005 & $0,8 \%$ & 2,5 & 0,14 & $6 \%$ & 2 & 0 & 0 \\
\hline 11 & 39,0 & 0,73 & 0,006 & $0,8 \%$ & 1,8 & 0,24 & $13 \%$ & 0 & 3 & 0 \\
\hline 12 & 30,0 & 0,61 & 0,005 & $0,8 \%$ & 2,0 & 0,18 & $9 \%$ & 1 & 0 & 4 \\
\hline 13 & 50,9 & 0,49 & 0,007 & $1,4 \%$ & 3,0 & 0,54 & $18 \%$ & 2 & 0 & 1 \\
\hline 14 & 33,5 & 0,31 & 0,009 & $2,9 \%$ & 4,4 & 0,62 & $14 \%$ & 2 & 0 & 0 \\
\hline 15 & 47,7 & 0,42 & 0,007 & $1,7 \%$ & 3,5 & 0,59 & $17 \%$ & 4 & 0 & 1 \\
\hline 16 & 30,0 & 0,64 & 0,005 & $0,8 \%$ & 2,0 & 0,42 & $21 \%$ & 0 & 0 & 2 \\
\hline 17 & 38,1 & 0,46 & 0,006 & $1,3 \%$ & 3,1 & 0,37 & $12 \%$ & 2 & 0 & 0 \\
\hline 18 & 45,0 & 0,59 & 0,008 & $1,3 \%$ & 2,9 & 0,44 & $15 \%$ & 3 & 0 & 0 \\
\hline 19 & 23,9 & 0,63 & 0,003 & $0,5 \%$ & 2,1 & 0,32 & $15 \%$ & 0 & 0 & 2 \\
\hline 20 & 29,1 & 0,50 & 0,007 & $1,4 \%$ & 2,6 & 0,49 & $19 \%$ & 2 & 0 & 0 \\
\hline
\end{tabular}

$\left(^{*}\right)$ B: bolted; O: overlapped cylinders; W: welded.

In all the tested sites, the damping measure is a global indicator of the energy dissipation ability of the monopole from all possible sources combined, also called the net damping ratio. Structural damping in the classical viscous model exponentially reduces the free vibration of a structure. However, damping is complex and not classical in these structures as it comes from a variety of sources: inherent structural damping (hysteretic) provided by constitutive materials and in welded joints, dry friction damping in bolted connections and at contact surfaces of nested cylinders in overlap joints, radiation damping provided by the soil surrounding foundations, and aerodynamic damping depending on the structure's shape and its exposure to wind, where supported 
antennas and appurtenances modify the aeroelastic properties of the whole system. Aerodynamic damping is deemed positive in all cases (contributing to reducing the vibrations). The right portion provides information on the number and type of connections used in the monopoles, as this information proves useful in the interpretation of the damping results. Three connection types are used: bolted flange connections (B), overlapping of nested tubes $(0)$, and welded joints (W). In general, it is observed that bolted joints appear to provide more damping than welded joints, while overlapping connections seem to provide the least damping effects. Of course, the connections are not the only contributors, but they appear to be the most significant, notwithstanding the importance of the positive aerodynamic damping contributed by the dense antenna and accessory layouts. For example, the smallest fundamental modal damping value ( $1 \%$ average) is obtained at Site No. 4 (also the stiffest monopole) with one overlapped joint and one welded joint and a sparse antenna layout with very large void ratio that promotes low aerodynamic effects. Site No. 7 (the shortest monopole tested) has only one bolted joint but supports a rather dense array of antennas and appurtenances that contribute to increase aerodynamic damping, for a net estimated ratio of $4,7 \%$. It is also interesting to consider Site No. 3 (49 m height) with an average measured damping of $4.1 \%$ with three bolted joints and a very small antenna payload.

It is important to note that the damping values obtained from AVM tests are associated with very small displacements, and that testing methods involving large motions would yield larger damping values. Our results appear consistent with those obtained by Pagnini \& Solari [9] who have conducted free-vibration release tests on tall luninaires. We have found no direct measures of aerodynamic damping alone for such structures, but numerical experiments on masts with circular cylindrical shapes [10] have indicated possible variations in the range of $0,5 \%$ to $2 \%$ maximum depending on wind speed. As for possible negative aerodynamic damping values associated with vortex shedding or wake-induced flutter in circular structures, no experimental results could be found in the literature but a value of $-0,5 \%$ is deemed conservative. Demartino \& Ricciardelli [4] present a review of the aerodynamics of nominally circular cylinders as determined by experiments but do not quote any aerodynamic damping values. Likewise, in [11] that deals specifically with aerodynamic forces on complex lighting poles and antenna masts.

\section{Conclusion}

This study confirms that ambient vibration tests conducted at and near the base of telecommunications monopoles provide reliable estimates of the fundamental frequencies and corresponding net modal damping. A statistical analysis of frequency estimates indicates small dispersion of the results, with a standard deviation not exceeding 3,3\% for the 20 tested structures. The uncertainty is expected to be larger for modal damping estimates considering the complexity of energy dissipation modes in such structures, and values between 1,0 and 4,7\% are obtained, with expected variability in the order of $20 \%$.

\section{Acknowledgement}

Financial support from the Natural Sciences and Engineering Research Council of Canada (NSERC) and from the Fonds de recherche du Québec Nature et Technologie, through the Interuniversity Center for the Study of Structures under Extreme Loadings (CEISCE) is greatly acknowledged. Thanks, and also due to WSP Consultants (Telecommunications) in Montreal for providing access to the test sites and delegating field engineers to accompany our test team.

\section{Conflict of Interest}

No conflict of interest.

\section{References}

1. CAN/CSA S37-18 (2018) Antennas, Towers and Antenna-Supporting Structures. Canadian Standards Association, Mississauga, Ontario, Canada.

2. EIA/TIA-222-H (2018) ANSI/TIA-222 Structural Standard for Antenna Supporting Structures, Antennas and Small Wind Turbine Support Structures. Telecommunication Industry Association.

3. MoHo srl (2019) TROMINO c/o VEGA, edificio Lybra, Via delle Industrie 17/A, 30175 Marghera (Venice), Italy.

4. Structural Vibration Solutions (2019) ARTeMIS Modal Standard Software. NOVI Science Park, Niels Jernes Vej 10, DK-9220 Aalborg East, Denmark.

5. Pastor M, Binda M, Harčarik T (2012) Modal assurance criterion. Procedia Engineering 48: 543-548.

6. Gade S, Møller NB, Herlufsen H, Konstantin-Hansen H (2005) Frequency domain techniques for operational modal analysis. Proc. $1^{\text {st }}$ International Operational Modal Analysis Conference (IOMAC 2005), 26-27 April 2005, Copenhagen, Denmark.

7. Foti D, Diaferio M, Giannoccaro NI, Mongelli M (2012) Ambient vibration testing, dynamic identification and model updating of a historic tower. NDT \& E International 47: 88-95.

8. Magalhães F, Brincker R, Cunha Á (2007) Damping Estimation Using Free Decays and Ambient Vibration Tests. Proc. $2^{\text {nd }}$ International Operational Modal Analysis Conference (IOMAC 2007), April 30 - May 2, 2007, Copenhagen, Denmark 2: 513-521.

9. Pagnini LC, Solari G (2001) Damping measurements of steel poles and tubular towers, Engineering Structures 23(9): 1085-1095.

10. Kallesøe BS, Sørensen NN, Troldborg N (2011) Aerodynamic damping of lateral tower vibrations. In M. H. Hansen (Ed.), Presentations from the Aeroelastic Workshop-latest results from AeroOpt Roskilde: Danmarks Tekniske Universitet, Risø Nationallaboratoriet for Bæredygtig Energi. Denmark. Forskningscenter Risoe. Risoe-R, No. 1796(EN).

11. Nguyen CH, Freda A, Solari G, Tubino F (2015) Aeroelastic instability and wind-excited response of complex lighting poles and antenna masts. Engineering Structures 85: 264-276. 